PROCEEDINGS OF THE

AMERICAN MATHEMATICAL SOCIETY

Volume 130, Number 4, Pages 1189-1195

S 0002-9939(01)06138-X

Article electronically published on October 1, 2001

\title{
ON PERFECTLY MEAGER SETS
}

\author{
TOMEK BARTOSZYNSKI
}

(Communicated by Alan Dow)

\begin{abstract}
We show that it is consistent that the product of perfectly meager sets is perfectly meager.
\end{abstract}

\section{INTRODUCTION}

Suppose that $X$ is a subset of a Polish space $\mathbf{X}$. We say that $X$ is perfectly meager if for every perfect set $P \subseteq \mathbf{X}, X \cap P$ is meager in the relative topology of $P$. Let $\mathbf{P M}$ denote the collection of perfectly meager sets.

Clearly all countable sets are perfectly meager but there are various examples of uncountable perfectly meager sets that can be constructed in ZFC (see [5]).

In [4, Marczewski asked whether the product of perfectly meager sets is perfectly meager. This question was partially answered by Recław who showed that:

Theorem 1 (Recław [7]). Assume $\mathrm{CH}$. Then there are two perfectly meager sets whose product is not perfectly meager.

The proof relies on the existence of a Borel set having certain properties and the existence of a Luzin set (i.e. an uncountable set whose intersection with every meager set is countable).

The purpose of this note is to show that it is also consistent with ZFC that the product of any two perfectly meager sets is perfectly meager. Thus, Marczewski's question is undecidable in ZFC.

Definition 2. Suppose that $X$ is a subset of a Polish space $\mathbf{X}$. We say that $X$ is universally meager if every Borel isomorphic image of $X$ in $\mathbf{X}$ is meager. Let $\mathbf{U M}$ denote the collection of universally meager sets.

It is clear that $\mathbf{U M} \subseteq \mathbf{P M}$, but the other inclusion may fail.

Theorem 3 (Sierpiński [8]). Assume $\mathbf{C H}$. Then $\mathbf{P M} \neq \mathbf{U M}$.

Unlike PM, the class of universally meager sets is closed under products.

Theorem 4 (Zakrzewski [10]). The product of universally meager sets is universally meager.

This is a consequence of the following characterization of the class UM. Let $\mathbb{C}$ denote the Cohen algebra.

Received by the editors May 5, 2000 and, in revised form, October 24, 2000.

1991 Mathematics Subject Classification. Primary 03E17.

Key words and phrases. Perfectly meager, products, consistency.

The author was partially supported by NSF grant DMS 99271.

(C)2001 American Mathematical Society 
Theorem 5 (Zakrzewski [10]). For a subset $X$ of a perfect Polish space $\mathbf{X}$, the following are equivalent:

(1) $X \in \mathbf{U M}$.

(2) $X$ does not contain a Borel one-to-one image of a non-meager set.

(3) For every $\sigma$-ideal $\mathcal{J}$ in $\operatorname{Borel}(\mathbf{X})$ such that $\operatorname{Borel}(\mathbf{X}) / \mathcal{J} \cong \mathbb{C}$ there is a Borel set $B \in \mathcal{J}$ such that $X \subseteq B$.

(4) $X$ is meager in every Polish topology $\tau$ on $\mathbf{X}$ such that $\mathbf{X}$ has no isolated points and $\operatorname{Borel}(\mathbf{X}, \tau)=\operatorname{Borel}(\mathbf{X})$.

(5) $X$ is meager in every second countable Hausdorff topology $\tau$ on $\mathbf{X}$ such that $\mathbf{X}$ has no isolated points and all Borel sets (in the original Polish topology) have Baire Property in the topology $\tau$.

(6) There is no $\sigma$-ideal $\mathcal{J}$ in $\operatorname{Borel}(X)$ such that $\operatorname{Borel}(X) / \mathcal{J} \cong \mathbb{C}$.

(7) $X$ is meager in every second countable Hausdorff topology $\tau$ on $X$ such that $X$ has no isolated points and all Borel subsets of $X$ (in the topology inherited from the original Polish topology on $\mathbf{X}$ ) have Baire Property in the topology $\tau$.

(8) $X$ is meager in every separable metrizable topology $\tau$ on $X$ such that $X$ has no isolated points and $\operatorname{Borel}(X)=\operatorname{Borel}(X, \tau)$.

Now we can formulate our main result:

Theorem 6. It is consistent that $\mathbf{P M}=\mathbf{U M}$. In particular, it is consistent that $\mathbf{P M}$ is closed under products.

\section{IN ZFC}

In this section we will identify a more general property that implies that $\mathbf{P M}=$ UM.

For a function $f: X \longrightarrow Y$ let $f[X]=\{f(x): x \in X\}$ denote the image of $X$.

We need the following observation:

Lemma 7. Suppose that $X \notin \mathbf{U M}$. Then there exists $X^{\prime} \subseteq X$, a Borel set $A \supseteq X^{\prime}$ and Borel isomorphism $f: A \longrightarrow \omega^{\omega}$ such that:

(1) $f^{-1}$ is continuous,

(2) $f\left[X^{\prime}\right]$ is not meager in $\omega^{\omega}$.

Proof. Assume that $X$ is a subset of a Polish space $\mathbf{X}$. Let $h: X \longrightarrow h[X]$ be a Borel isomorphism witnessing that $X \notin \mathbf{U M}$. Find Borel sets $A^{\prime}, B \subseteq \mathbf{X}, A^{\prime} \supseteq X$ and $B \supseteq h[X]$ and the Borel isomorphism $\bar{h}: A^{\prime} \longrightarrow B$ extending $h$ (exercise in [9]).

Apply Kuratowski's theorem $\left([\underline{3}, 8.38)\right.$ to get a dense $G_{\delta}$ subset $B^{\prime} \subseteq B$ such that $\bar{h}^{-1} \nmid B$ is continuous. Since $B^{\prime}$ is a nonmeager Borel set it contains a relative $G_{\delta}$ dense subset $B^{\prime \prime}$ which is homeomorphic to $\omega^{\omega}$ via a homeomorphism $g$. Finally set $A=\bar{h}^{-1}\left(B^{\prime \prime}\right)$ and $f=g \circ \bar{h}$, and notice that this works since $\bar{h}[X] \cap B^{\prime \prime}$ is not meager in $B^{\prime \prime}$.

Consider the following principle:

Definition 8. Axiom P: For every nonmeager subset $X \subseteq \omega^{\omega}$ there exists a compact subset $P \subseteq \omega^{\omega}$ such that $P \cap X$ is nonmeager in $P$.

Theorem 9. Assume P. Then $\mathbf{U M}=\mathbf{P M}$. 
Proof. Suppose that $X \notin \mathbf{U M}$. By Lemma 7 there exists $X^{\prime} \subseteq X$, a Borel set $A \supseteq X^{\prime}$ and Borel isomorphism $f: A \longrightarrow \omega^{\omega}$ such that:

(1) $f^{-1}$ is continuous,

(2) $f\left[X^{\prime}\right]$ is not meager in $\omega^{\omega}$.

Apply Axiom $\mathrm{P}$ to to find a compact set $P \subseteq \omega^{\omega}$ such that $P \cap f\left[X^{\prime}\right]$ is not meager in $P$. Set $Q=f^{-1}(P)$ and note that $f\lceil Q$ is a homeomorphism between $P$ and $Q$. Under this homeomorphism $Q \cap X^{\prime}$ is the image of $f\left[X^{\prime}\right] \cap P$, thus it is not meager in $Q$. Since $Q \cap X \supseteq Q \cap X^{\prime}$ it follows that $X \notin \mathbf{P M}$. As the other inclusion is obvious, the theorem follows.

\section{FORCING}

In this section we will show that Axiom $\mathrm{P}$ is consistent with ZFC, which will finish the proof.

For a tree $p$ and $t \in p$, let $\operatorname{succ}_{p}(t)$ be the set of all immediate successors of $t$ in $p, p_{t}=\{v \in p: t \subseteq v$ or $v \subseteq t\}$ the subtree of $p$ determined by $t$, and let $[p]$ be the set of branches of $p$. By identifying $s \in \omega^{<\omega}$ with the full-branching tree having root $s$, we can also denote $[s]=\left\{f \in \omega^{\omega}: s \subseteq f\right\}$. Let $\omega^{\uparrow \omega}=\left\{s \in \omega^{<\omega}\right.$ : $s$ is strictly increasing .

The rational perfect forcing $\mathbb{M}$ is the following forcing notion:

$p \in \mathbb{M} \Longleftrightarrow p \subseteq \omega^{\uparrow \omega}$ is a perfect tree \&

$$
\forall s \in p \exists t \in p\left(s \subseteq t \&\left|\operatorname{succ}_{p}(t)\right|=\aleph_{0}\right) .
$$

For $p, q \in \mathbb{M}, p \geq q$ if $p \subseteq q$. Without loss of generality we can assume that $\left|\operatorname{succ}_{p}(s)\right|=1$ or $\left|\operatorname{succ}_{p}(s)\right|=\aleph_{0}$ for all $p \in \mathbb{M}$ and $s \in p$. Conditions of this type form a dense subset of $\mathbb{M}$.

Let

$$
\operatorname{split}(p)=\left\{s \in p:\left|\operatorname{succ}_{p}(s)\right|>1\right\}=\bigcup_{n \in \omega} \operatorname{split}_{n}(p),
$$

where $\operatorname{split}_{n}(p)=\{s \in \operatorname{split}(p):|\{t \subsetneq s: t \in \operatorname{split}(p)\}|=n\}$.

For $p, q \in \mathbb{M}, n \in \omega$, we let

$$
p \geq_{n} q \Longleftrightarrow p \geq q \& \operatorname{split}_{n}(q)=\operatorname{split}_{n}(p) .
$$

If $v \in \operatorname{split}(p)$ let $U_{v}^{p}=\left\{n \in \omega: v^{\frown} n \in p\right\}$ and for $n \in U_{v}^{p}$ let $v^{n}$ be the first splitting node below $v^{\frown} n$.

If $G \subseteq \mathbb{M}$ is a generic filter over $\mathbf{V}$ let $\mathbf{m}=\bigcap_{p \in G}[p]$ be the generic real.

Let $\mathbb{M}_{\omega_{2}}$ be the countable support iteration of $\mathbb{M}$ of length $\aleph_{2}$.

The following facts about $\mathbb{M}$ are well-known.

Theorem 10. (1) The sequence $\left\langle\leq_{n}: n \in \omega\right\rangle$ witnesses that $\mathbb{M}$ satisfies axiom A.

In particular, $\mathbb{M}$ is proper.

(2) $\mathbb{M}$ preserves non-meager sets, i.e. if $A \subseteq 2^{\omega}$, where $A \in \mathbf{V}$ is not meager, then $\mathbf{V}^{\mathbb{M}}=A$ is not meager ([1], Theorem 7.3.46).

(3) $\mathbb{M}$ also satisfies the iterable condition for preserving non-meager sets. In particular, countable support iteration of Miller forcing preserves non-meager sets ([1], Theorems 6.3.19 and 6.3.20). 
Theorem 11. $\mathrm{V}^{\mathbb{M}_{\omega_{2}}} \models$ Axiom $\mathrm{P}$.

The idea of the proof is as follows. Suppose that $X \in \mathbf{V}^{\mathbb{M}_{\omega_{2}}}$ and $X$ is not meager in $\omega^{\omega}$. First we find $\alpha<\omega_{2}$ such that $\mathbf{V}^{\mathbb{M}_{\alpha}} \models X \cap \mathbf{V}^{\mathbb{M}_{\alpha}}$ is not meager (Lemma 12). Next we will find a compact set $P \subseteq \omega^{\omega}$ belonging to $\mathbf{V}^{\mathbb{M}_{\alpha+1}}$ such that

$$
\mathbf{V}^{\mathbb{M}_{\alpha+1}} \models P \cap X \cap \mathbf{V}^{\mathbb{M}_{\alpha}} \text { is not meager in } P \text { (Theorem [13). }
$$

Finally, by $10(3), \mathbb{M}_{\omega_{2}}$ preserves non-meager sets. Thus

$$
\mathbf{V}^{\mathbb{M} \omega_{2}} \models X \cap \mathbf{V}^{\mathbb{M} \alpha} \text { is not meager in } P \text {, }
$$

which implies that $\mathbf{V}^{\mathbb{M}_{\omega_{2}}} \models X$ is not meager in $P$.

Lemma 12. Suppose that $X \in \mathbf{V}^{\mathbb{M}_{\omega_{2}}}, X \subseteq \omega^{\omega}$ and $X$ is not meager in $\mathbf{V}^{\mathbb{M}_{\omega_{2}}}$. Then there exists $\alpha<\omega_{2}$ such that $\mathbf{V}^{\mathbb{M}_{\alpha}} \models X \cap \mathbf{V}^{\mathbb{M}_{\alpha}}$ is not meager.

Proof. Let $\left\langle\alpha_{\xi}: \xi<\omega_{1}\right\rangle$ be a continuous increasing sequence such that

$$
\mathbf{V}^{\mathbb{M}_{\omega_{2}}} \models X \cap \mathbf{V}^{\mathbb{M}_{\alpha_{\xi+1}}} \text { is not covered by any meager set from } \mathbf{V}^{\mathbb{M}_{\alpha_{\xi}}} \text {. }
$$

By properness, $\alpha=\sup _{\xi<\omega_{1}} \alpha_{\xi}$ has the required property, because every real in $\mathbf{V}^{\mathbb{M}_{\alpha}}$ is in some $\mathbf{V}^{\mathbb{M}_{\xi}}$.

Theorem 13. Suppose that $X \in \mathbf{V}, X \subseteq \omega^{\omega}$ is a non-meager set. There is a compact set $P \subseteq \omega^{\omega}, P \in \mathbf{V}^{\mathbb{M}}$ such that $: \overline{\mathbf{V}}^{\mathbb{M}} \models X$ is not meager in $P$.

Proof. For the sake of clarity we will break the proof into three lemmas. The main idea of the proof is already present in [2].

Let $\dot{\mathbf{m}}$ be the canonical name for an $\mathbb{M}$-generic real and let $\mathbb{C}$ be the Cohen forcing represented as $\omega^{<\omega}$ with $\dot{\mathbf{c}}$ being the canonical name for the Cohen real.

For $x \in \omega^{\omega}$ let $P_{x}=\left\{z \in \omega^{\omega}: \forall n z(n) \leq x(n)\right\}$. Note that $P_{x}$ is a compact set in $\omega^{\omega}$.

For two sequences $s \in \omega^{<\omega}, t \in \omega^{\uparrow \omega}$ we say that $(s, t)$ is $\operatorname{good}$ if $|s|=|t|$ and $s(i) \leq t(i)$ for $i<|t|$.

Lemma 14. Suppose that $p \in \mathbb{M}, s \in \mathbb{C}$, and $(s|| \operatorname{stem}(p) \mid$, stem $(p))$ is good. Then there is a $\mathbb{C}$-name $\dot{q}$ for an element of $\mathbb{M}$ such that:

(1) $s \Vdash_{\mathbb{C}} \dot{q} \geq_{0} p$,

(2) $(s, \dot{q}) \Vdash_{\mathbb{C} \star \dot{\mathbb{M}}} \dot{\mathbf{c}} \in P_{\dot{\mathbf{m}}}$.

Proof. Let $\mathbf{c} \supseteq s$ be a Cohen real over $\mathbf{V}$. Working in $\mathbf{V}[\mathbf{c}]$ define

$$
q=\{v \in p:(\mathbf{c}\lceil|v|, v) \text { is good }\} .
$$

It is enough to check that $q \in \mathbb{M}^{\mathbf{V}[\mathbf{c}]}$. In fact, we will show that if $v \in \operatorname{split}(p)$ and $v \in q$, then $v \in \operatorname{split}(q)$.

Suppose that $v \in \operatorname{split}(p)$ and $v \in q$. In particular, $(\mathbf{c}|| v \mid, v)$ is good. Let $s^{\prime}=\mathbf{c} \uparrow|v|$. For $k \in \omega$ let

$$
D_{k}=\left\{t \in \mathbb{C}: s^{\prime} \subseteq t \& \exists n>k\left(t \Uparrow\left|v^{n}\right|, v^{n}\right) \text { is good }\right\} .
$$

We show that $D_{k}$ is dense in $\mathbb{C}$ below $s^{\prime}$. Take any $s^{\prime \prime} \geq s^{\prime}$ and let $n \in U_{v}^{p} \backslash$ $\max \left(\operatorname{range}\left(s^{\prime \prime}\right), k\right)$. If $\left|s^{\prime \prime}\right|>\left|v^{n}\right|$, then put $t=s^{\prime \prime}$. Otherwise, let $t \geq s^{\prime \prime}$ be such that $|t|=\left|v^{n}\right|$ and

$$
t(i)= \begin{cases}s^{\prime \prime}(i) & \text { if } i<\left|s^{\prime \prime}\right| \\ 0 & \text { if }\left|s^{\prime \prime}\right| \leq i<\left|v^{n}\right|\end{cases}
$$


It is clear that $t \in D_{k}$. By genericity, we conclude that the set

$$
\left\{n:\left(\mathbf{c}|| v^{n} \mid, v^{n}\right) \text { is good }\right\}
$$

is infinite in $\mathbf{V}[\mathbf{c}]$. In particular, $v \in \operatorname{split}(q)$. Since $\mathbf{c}$ was arbitrary, it finishes the proof.

Lemma 15. Suppose that $p \in \mathbb{M}, s \in \mathbb{C}$, and $(s\rceil|\operatorname{stem}(p)|$, stem $(p))$ is good. Let $\dot{F}$ be an $\mathbb{M}$-name for a closed nowhere dense subset of $P_{\mathbf{m}}$. There exists a $\mathbb{C}$-name $\dot{q}$ for an element of $\mathbb{M}$ such that:

(1) $s \Vdash_{\mathbb{C}} \dot{q} \geq_{0} p$,

(2) $(s, \dot{q}) \Vdash_{\mathbb{C} \star \dot{M}} \dot{\mathbf{c}} \in P_{\mathbf{m}}$,

(3) $(s, \dot{q}) \Vdash_{\mathbb{C} \star \dot{\mathbb{M}}} \dot{\mathbf{c}} \notin \dot{F}$.

Proof. Let $\mathbf{m} \in[p]$ be an $\mathbb{M}$-generic real over $\mathbf{V}$, and let $F$ be the interpretation of $\dot{F}$ using $\mathbf{m}$. In $\mathbf{V}[\mathbf{m}]$ define sequences $\left\langle s_{n}: n \in \omega\right\rangle \in\left(\omega^{<\omega}\right)^{\omega}$ such that:

(1) $\forall v \in \prod_{j<n}(\mathbf{m}(j)+1)\left[v \frown s_{n}\right] \cap F=\emptyset$,

(2) $s_{n} \in \prod_{j=n}^{k}(\mathbf{m}(j)+1)$ for some $k>n$.

Since $F$ is nowhere dense this definition is correct. Going back to $\mathbf{V}$ we conclude that there is an $\mathbb{M}$-name $\left\langle\dot{s}_{n}: n \in \omega\right\rangle$ such that:

(1) $p \Vdash_{\mathbb{M}} \forall v \in \prod_{j<n}(\dot{\mathbf{m}}(j)+1)\left[v \frown \dot{s}_{n}\right] \cap \dot{F}=\emptyset$,

(2) $p \Vdash_{\mathbb{M}} \forall n\left(\dot{s}_{n}, \dot{\mathbf{m}}\right\rceil\left[n, n+\left|\dot{s}_{n}\right|\right)$ is good.

For each $n \in U_{\text {stem }(p)}^{p}$ find a condition $p_{n} \geq p$ such that:

(1) there is a sequence $s_{n}$ such that $p_{n} \Vdash_{\mathbb{M}} \dot{s}_{n}=s_{n}$,

(2) $\operatorname{stem}\left(p_{n}\right) \supseteq \operatorname{stem}(p)^{n}$,

(3) $\left|\operatorname{stem}\left(p_{n}\right)\right| \geq n+\left|s_{n}\right|$.

Observe that by the choice of $\left\langle\dot{s}_{n}: n \in \omega\right\rangle$ it follows that

$$
\left(s_{n}, \operatorname{stem}\left(p_{n}\right)\left\lceil\left[n, n+\left|s_{n}\right|\right)\right. \text { is good. }\right.
$$

Let $\mathbf{c} \supseteq s$ be a Cohen real over $\mathbf{V}$. Working in $\mathbf{V}[\mathbf{c}]$ define

$$
A=\left\{n \in \omega:\left(\mathbf{c} \uparrow\left|\operatorname{stem}\left(p_{n}\right)\right|, \operatorname{stem}\left(p_{n}\right)\right) \text { is good and } \mathbf{c} \uparrow\left[n, n+\left|s_{n}\right|\right)=s_{n}\right\} .
$$

We will show that $A$ is an infinite set in $\mathbf{V}[\mathbf{c}]$.

For $k \in \omega$ let

$$
\begin{aligned}
D_{k}=\left\{t \in \mathbb{C}: s \subseteq t \& \exists n>k\left(\left(t|| \operatorname{stem}\left(p_{n}\right) \mid, \operatorname{stem}\left(p_{n}\right)\right)\right.\right. & \text { is good \& } \\
& \left.\left.t \uparrow\left[n, n+\left|s_{n}\right|\right)=s_{n}\right)\right\} .
\end{aligned}
$$

We show that $D_{k}$ is dense in $\mathbb{C}$ below $s$. Suppose that $s^{\prime} \geq s$ and let $\ell=$ $\max \left(\operatorname{range}\left(s^{\prime}\right),\left|s^{\prime}\right|, k\right)$. Pick $n \in U_{\text {stem }(p)}^{p} \backslash \ell$ and define $t \geq s^{\prime}$ such that $|t|=$ $\left|\operatorname{stem}\left(p_{n}\right)\right|$ and

$$
t(i)= \begin{cases}s^{\prime}(i) & \text { if } i<\left|s^{\prime}\right|, \\ 0 & \text { if }\left|s^{\prime}\right| \leq i<n, \\ s_{n}(i) & \text { if } n \leq i<n+\left|s_{n}\right|, \\ 0 & \text { if } n+\left|s_{n}\right| \leq i<\left|\operatorname{stem}\left(p_{n}\right)\right| .\end{cases}
$$

Note that by the properties of $\left\langle\dot{s}_{n}: n \in \omega\right\rangle$ it follows that $t \in D_{k}$. By genericity, for every $k \in \omega$ there is $n \geq k$ such that $\mathbf{c}\left\lceil n \in D_{k}\right.$, which implies that $A$ is infinite. 
Let $p^{\star}=\bigcup_{n \in U_{\text {stem }(p)}^{p}} p_{n}$. Define in $\mathbf{V}[\mathbf{c}]$,

$$
q_{1}=\bigcup_{n \in A} p_{n} \quad \text { and } \quad q_{2}=\left\{v \in p^{\star}:(\mathbf{c} \uparrow|v|, v) \text { is good }\right\},
$$

and let $q=q_{1} \cap q_{2}$. Since the nodes corresponding to the elements of $A$ were good, by Lemma 14, it follows that $q \in \mathbb{M}^{\mathbf{V}[\mathbf{c}]}$ and $q \geq_{0} p$. In addition, in $\mathbf{V}[\mathbf{c}]$, $q \Vdash_{\mathbb{M}} \forall n \mathbf{c}(n) \leq \dot{\mathbf{m}}(n)$ (by the choice of $q_{2}$ ) and $q \Vdash_{\mathbb{M}} \mathbf{c} \notin \dot{F}$ (by the choice of $q_{1}$ ). Since $\mathbf{c}$ was arbitrary, the proof is finished.

Finally we show:

Lemma 16. Suppose that $p \in \mathbb{M}, s \in \mathbb{C}$, and $(s \uparrow|\operatorname{stem}(p)|, \operatorname{stem}(p))$ is good. Let $\left\langle\dot{F}_{n}: n \in \omega\right\rangle$ be an $\mathbb{M}$-name for a sequence of closed nowhere dense subsets of $P_{\dot{\mathbf{m}}}$. There exists a $\mathbb{C}$-name $\dot{q}$ for an element of $\mathbb{M}$ such that:

(1) $s \Vdash_{\mathbb{C}} \dot{q} \geq p$,

(2) $(s, \dot{q}) \Vdash_{\mathbb{C} \star \dot{M} \mathbb{M}} \dot{\mathbf{c}} \in P_{\dot{m}}$,

(3) $(s, \dot{q}) \Vdash_{\mathbb{C} \star \dot{\mathbb{M}}} \dot{\mathbf{c}} \notin \bigcup_{n} \dot{F}_{n}$.

Proof. The proof is a refinement of the proof of the previous lemma. Suppose that $\left\langle\dot{F}_{n}: n \in \omega\right\rangle$ is an $\mathbb{M}$-name for a sequence of closed nowhere dense subsets of $P_{\mathbf{m}}$. Without loss of generality we can assume that $\Vdash_{\mathbb{M}} \forall n \dot{F}_{n} \subseteq \dot{F}_{n+1}$. Find an $\mathbb{M}$-name $\left\langle\dot{s}_{n}: n \in \omega\right\rangle$ such that:

(1) $p \Vdash_{\mathbb{M}} \forall v \in \prod_{j<n}(\dot{\mathbf{m}}(j)+1)\left[v \frown \dot{s}_{n}\right] \cap \dot{F}_{n}=\emptyset$,

(2) $p \Vdash_{\mathbb{M}} \forall n\left(\dot{s}_{n}, \dot{\mathbf{m}}\left\lceil\left[n, n+\left|\dot{s}_{n}\right|\right)\right.\right.$ is good.

Build by induction a sequence of conditions $\left\langle p_{n}: n \in \omega\right\rangle$ such that:

(1) $p_{0}=p$,

(2) $p_{n+1} \geq_{n} p_{n}$

(3) if $v \in \operatorname{split}_{n}\left(p_{n+1}\right)$ and $k \in U_{v}^{p_{n+1}}$, then there exists a sequence $s_{v, k} \in \omega^{<\omega}$ such that:
(a) $\left(p_{n+1}\right)_{v^{k}} \Vdash_{\mathbb{M}} \dot{s}_{k}=s_{v, k}$,
(b) $\left|v^{k}\right| \geq k+\left|s_{v, k}\right|$

As in the previous lemma, it follows that for $v \in \operatorname{split}_{n}\left(p_{n+1}\right)$ and $k \in U_{v}^{p_{n+1}}$, $\left(s_{v, k}, v^{k} \uparrow\left[k, k+\left|s_{v, k}\right|\right)\right)$ is good.

The construction is straightforward; the first step is essentially described in the previous lemma. Let $p^{\star}=\bigcap_{n} p_{n}$ and let $\mathbf{c} \supseteq s$ be a Cohen real over V. Working in $\mathbf{V}[\mathbf{c}]$ define for each $n \in \omega$ and $v \in \operatorname{split}_{n}\left(p^{\star}\right)$ :

$$
A^{v}=\left\{k \in U_{v}^{p^{\star}} \backslash n:(\mathbf{c}\rceil\left|v^{k}\right|, v^{k}\right) \text { is good and } \mathbf{c}\left\lceil\left[k, k+\left|s_{v, k}\right|\right)=s_{v, k}\right\} .
$$

As before, it follows that $A^{v}$ is infinite in $\mathbf{V}[\mathbf{c}]$ for every $v \in \operatorname{split}\left(p^{\star}\right)$. Finally, let $q \geq p^{\star} \geq p$ be defined so that for every $v \in \operatorname{split}(q), U_{v}^{q}=A^{v}$.

It follows from the definition of $q$ that $\mathbf{V}[\mathbf{c}] \models q \Vdash_{\mathbb{M}} \mathbf{c} \in P_{\dot{\mathbf{m}}}$. On the other hand, for every $v \in \operatorname{split}_{n}(q), \mathbf{V}[\mathbf{c}] \models q_{v} \Vdash_{\mathbb{M}} \exists k>n \mathbf{c} \notin \dot{F}_{k}$. Thus

$$
\mathbf{V}[\mathbf{c}] \models q \Vdash_{\mathbb{M}} \exists^{\infty} n \mathbf{c} \notin \dot{F}_{n} .
$$

Since the sets $\dot{F}_{n}$ are increasing, we conclude that $\mathbf{V}[\mathbf{c}] \models q \Vdash_{\mathbb{M}} \mathbf{c} \notin \bigcup_{n} \dot{F}_{n}$.

Now we are ready to prove Theorem 13 Suppose that $X \in \mathbf{V}, X \subseteq \omega^{\omega}$ is not meager. We will show that $\Vdash_{\mathbb{M}} X \cap P_{\dot{\mathbf{m}}}$ is not meager in $P_{\mathbf{m}}$. Suppose otherwise 
and let $\left\langle\dot{F}_{n}: n \in \omega\right\rangle$ be an $\mathbb{M}$-name for a sequence of closed nowhere dense sets in $P_{\dot{m}}$ such that for some $p \in \mathbb{M}$,

$$
p \Vdash_{\mathbb{M}} X \cap P_{\dot{m}} \subseteq \bigcup_{n \in \omega} \dot{F}_{n} .
$$

Let $N \prec \mathbf{H}(\chi)$ be a countable elementary submodel containing $p, X,\left\langle\dot{F}_{n}: n \in\right.$ $\omega\rangle$, etc. Since $X$ is not meager there exists a real $\mathbf{c} \in X$ which is Cohen over $N$. By Lemma 16 there exists a condition $q \geq p, q \in N[\mathbf{c}]$ such that

$$
q \Vdash_{\mathbb{M}} \mathbf{c} \in P_{\dot{\mathbf{m}}} \backslash \bigcup_{n \in \omega} \dot{F}_{n} .
$$

This contradicts the choice of $p$ and finishes the proof.

Note that in fact we have showed the following:

Theorem 17. Suppose that $\left\langle\mathcal{P}_{\alpha}, \dot{\mathcal{Q}}_{\alpha}: \alpha<\omega_{2}\right\rangle$ is a countable support iteration of proper forcing notions such that:

(1) $\left\{\alpha<\omega_{2}: \Vdash_{\alpha} \dot{\mathcal{Q}}_{\alpha} \simeq \mathbb{M}\right\}$ is cofinal in $\aleph_{2}$,

(2) $\Vdash_{\alpha} \dot{\mathcal{Q}}_{\alpha}$ preserves non-meager sets for $\alpha<\omega_{2}$.

Then $\mathbf{V}^{\mathcal{P}_{\omega_{2}}} \models$ Axiom $\mathbf{P}$.

\section{ACKNOWLEDGEMENTS}

The work was done while I was spending a sabbatical year at Rutgers University and the College of Staten Island, CUNY, and I thank their mathematics departments for the support. I am also grateful to Andrzej Rosłanowski for carefully proofreading earlier versions of this paper and giving many valuable suggestions.

\section{REFERENCES}

[1] Tomek Bartoszyński and Haim Judah. Set Theory: on the structure of the real line. A.K. Peters, 1995. MR 96k:03002

[2] Haim Judah and Saharon Shelah. Killing Luzin and Sierpiński sets. Proceedings of the American Mathematical Society, 120:917-920, 1994. MR 94e:03046

[3] Alexander Kechris. Classical Descriptive Set Theory, volume 156 of Graduate Texts in Mathematics. Springer Verlag, 1995. MR 96e:03057

[4] Edward Marczewski (Szpilrajn). Probleme 68. Fundamenta Mathematicae, 25:579, 1935.

[5] Arnold W. Miller. Special subsets of the real line. In K. Kunen and J. E. Vaughan, editors, Handbook of Set Theoretic Topology, pages 201-235. North-Holland, Amsterdam, 1984. MR 86i:54037

[6] Janusz Pawlikowski. Products of perfectly meager sets and Lusin's function. Proceedings of the American Mathematical Society, 107(3):811-815, 1989. MR 90b:54025

[7] Ireneusz Recław. Products of perfectly meagre sets. Proceedings of the American Mathematical Society, 112(4):1029-1031, 1991. MR 91j:28001

[8] Waclaw Sierpiński. Sur une probleme de M.Kuratowski concernant la propriete de Baire des ensambles. Fundamenta Mathematicae, 22:262-266, 1934.

[9] S.M Srivastava. A Course on Borel sets. Graduate Texts in Mathematics. Springer-Verlag, 1998. MR 99d:04002

[10] Piotr Zakrzewski. Universally meager sets. to appear in Proceedings of the American Mathematical Society.

Department of Mathematics and Computer Science, Boise State University, Boise, IDAHO 83725

E-mail address: tomek@math.boisestate.edu

$U R L:$ http://math.boisestate.edu/ ${ }^{\sim}$ tomek 